\title{
Diagnostic value of neutrophil gelatinase-associated lipocalin for renal injury in asphyxiated preterm infants
}

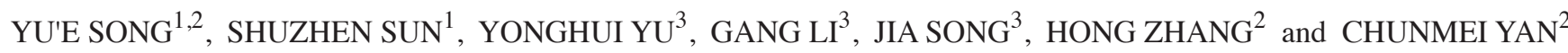 \\ ${ }^{1}$ Department of Pediatric Nephrology and Rheumatism and Immunology, Shandong Provincial Hospital Affiliated \\ to Shandong University, Jinan, Shandong 250021; ${ }^{2}$ Department of Pediatrics, The First Affiliated Hospital of \\ Baotou Medical College, Baotou, Inner Mongolia 014010; ${ }^{3}$ Department of Neonatology, \\ Shandong Provincial Hospital Affiliated to Shandong University, Jinan, Shandong 250021, P.R. China
}

Received April 14, 2016; Accepted September 12, 2016

DOI: $10.3892 /$ etm.2017.4107

\begin{abstract}
The aim of the present study was to analyze the diagnostic value of neutrophil gelatinase-associated lipocalin (NGAL) in renal injury in asphyxiated preterm infants. In total, 48 cases of asphyxiated preterm infants, 45 cases of premature infants and 45 cases of normal newborn infants were included in the study. Using ELISA we evaluated the level of urine NGAL, kidney injury molecule-1 (KIM-1), cystatin $\mathrm{C}$ (Cys-C) and serum creatinine (Scr). We also calculated the estimated glomerular filtration rate (eGFR). Our results showed that NGAL, KIM-1 and Cys-C levels in the group of asphyxiant renal injury within 24 and $48 \mathrm{~h}$ were markedly higher than the other two groups $(\mathrm{P}<0.05)$, while changes in Scr and eGFR within $24 \mathrm{~h}$ were not significant $(\mathrm{P}>0.05)$. Scr level in renal injury group within $48 \mathrm{~h}$ was markedly elevated while the eGFR level was visibly reduced $(\mathrm{P}<0.05)$. According to analysis of receiver operating characteristic curve, area under curve for NGAL in the group of asphyxiant renal injury within 24 and $48 \mathrm{~h}$ was significantly higher than KIM-1 and Cys-C. Susceptibility and specificity were improved. In conclusion, diagnosis of renal injury in asphyxia neonates using NGAL was more efficient compared to other methods.
\end{abstract}

\section{Introduction}

Renal injury in asphyxiated preterm infants has a high incidence rate $(30-55 \%)$ and the mortality rate is $60-66 \%$ (1). According to the definition of acute kidney injury (AKI) and classification of RIFLE (2) which was presented by AKI network (AKIN) in

Correspondence to: Dr Shuzhen Sun, Department of Pediatric Nephrology and Rheumatism and Immunology, Shandong Provincial Hospital Affiliated to Shandong University, 324 Jingwu Road, Jinan, Shandong 250021, P.R. China

E-mail: sun_sz1@163.com

Key words: neutrophil gelatinase-associated lipocalin, renal injury of asphyxia preterm infant, kidney injury molecule-1, cystatin C
2007, the likelihood of diagnosing renal injury in asphyxiated preterm infants was poor (3). Results obtained from at least one study showed that neutrophil gelatinase-associated lipocalin (NGAL), kidney injury molecule-1 (KIM-1) and level of cystatin C (Cys-C) could be used as sensitive biochemical markers for diagnosing renal injury in the early stages (4).

The same study showed that NGAL, KIM-1 and Cys-C in vivo levels were higher and more stable compared to serum creatinine (Scr) and urea nitrogen levels in the early stages of the disease (4).

Through comparative analysis, it was shown that these three indexes (NGAL, KIM-1 and Cys-C) had peak changes at different time-points and were closely related to the degree of renal injury $(5,6)$. The present study explored the significance of using NGAL for diagnosing renal injury in asphyxiated preterm infants and whether NGAL had a better diagnostic value.

\section{Materials and methods}

Patients. From October 2014 to October 2015, we enrolled 48 cases of asphyxiated preterm infants which were admitted to our hospital. We excluded cases with: i) Congenital monstrosity; ii) inherited metabolic diseases; iii) hypoxic ischemia encephalopathy; iv) severe asphyxia with no chance of recovery; and v) serious conditions with expected survival time of less than one month. There were 27 males and 21 females with an average age of 15.6 $\pm 7.2 \mathrm{~h}$ (range, 1-24 h). Average birth weight was $2.1 \pm 0.6 \mathrm{~kg}$ (range, $1.7-2.3 \mathrm{~kg}$ ).

Average gestational period was $35.6 \pm 2.4$ weeks (range, 34-37 weeks). We also enrolled 45 cases of premature infants without renal injury after asphyxia at the same time. Of these cases, there were 25 males and 20 females with an average age of $15.9 \pm 7.5 \mathrm{~h}$ (range, $1.5-28 \mathrm{~h}$ ). The average birth weight was $2.3 \pm 0.9 \mathrm{~kg}$ (range, $1.6-2.5 \mathrm{~kg}$ ), and the average gestational period was $35.9 \pm 2.6$ weeks (range, $35-37$ weeks).

Forty-five cases of normal newborn infants were also enrolled and of those there were 26 males and 19 females with an average age of $17.5 \pm 6.6 \mathrm{~h}$ (range, 2-30 h). The average birth weight for this group was $2.6 \pm 0.8 \mathrm{~kg}$ (range, 2.3-3.1 kg) Differences in gender, birth time, birth weight and gestational period for premature infants with renal injury and that of premature infants without renal injury had no statistical 
Table I. Comparison of observation index of each group.

\begin{tabular}{|c|c|c|c|c|c|c|c|c|c|c|}
\hline \multirow[t]{2}{*}{ Groups } & \multicolumn{5}{|c|}{$24 \mathrm{~h}$} & \multicolumn{5}{|c|}{$48 \mathrm{~h}$} \\
\hline & $\begin{array}{l}\text { NGAL } \\
(\mathrm{ng} / \mathrm{ml})\end{array}$ & $\begin{array}{c}\text { KIM-1 } \\
\text { (ng/l) }\end{array}$ & $\begin{array}{l}\text { Cys-C } \\
\text { (ng/mg) }\end{array}$ & $\begin{array}{c}\text { Scr } \\
(\mu \mathrm{mol} / 1)\end{array}$ & $\begin{array}{c}\text { eGFR } \\
(\mathrm{ml} / \mathrm{min})\end{array}$ & NGAL & KIM-1 & Cys-C & Scr & eGFR \\
\hline $\begin{array}{l}\text { Renal } \\
\text { injury }\end{array}$ & $55.8 \pm 15.4$ & $33.4 \pm 13.6$ & $23.6 \pm 10.8$ & $65.8 \pm 20.3$ & $105.8 \pm 23.5$ & $196.3 \pm 36.7$ & $76.7 \pm 25.3$ & $65.5 \pm 23.4$ & $194.6 \pm 42.5$ & $82.5 \pm 21.4$ \\
\hline $\begin{array}{l}\text { Premature } \\
\text { infant }\end{array}$ & $12.9 \pm 4.2$ & $7.5 \pm 1.4$ & $9.2 \pm 1.7$ & $62.3 \pm 24.5$ & $109.3 \pm 24.6$ & $15.7 \pm 5.3$ & $8.2 \pm 1.9$ & $9.7 \pm 1.5$ & $65.7 \pm 25.9$ & $103.2 \pm 29.7$ \\
\hline Normal & $10.6 \pm 4.9$ & $6.3 \pm 1.7$ & $8.7 \pm 1.3$ & $56.7 \pm 23.6$ & $114.5 \pm 23.8$ & $11.2 \pm 5.4$ & $6.9 \pm 1.6$ & $8.6 \pm 1.4$ & $53.4 \pm 22.3$ & $115.7 \pm 26.6$ \\
\hline F-value & 16.825 & 12.364 & 10.523 & 0.657 & 0.754 & 34.527 & 25.802 & 16.957 & 9.635 & 6.754 \\
\hline $\mathrm{P}$-value & $<0.001$ & $<0.001$ & $<0.001$ & 0.438 & 0.632 & $<0.001$ & $<0.001$ & $<0.001$ & $<0.001$ & $<0.001$ \\
\hline
\end{tabular}

NGAL, neutrophil gelatinase-associated lipocalin; KIM-1, kidney injury molecule-1; Cys-C, cystatin C; Scr, serum creatinine; eGFR, estimated glomerular filtration rate.

significance $(\mathrm{P}>0.05)$. Differences in gender and birth time in normal newborn infants group and that of the remaining two groups had no statistical significance, while birth weight was significantly higher.

The present study was approved by the Ethics Committee of Shandong Provincial Hospital. Written informed consent of the patients' guardians was obtained.

Detection index and methods. ELISA was used to detect NGAL, KIM-1, Cys-C and Scr levels in urine. The estimated glomerular filtration rate (eGFR) was calculated. Urine $(10 \mathrm{ml})$ and blood $(3 \mathrm{ml})$ specimens were collected in 24 and $48 \mathrm{~h}$. Specimens were preserved in low temperature after centrifugation $(2,500 \mathrm{x} \mathrm{g}$ for $5 \mathrm{~min})$, and then sent to the laboratory. NGAL, KIM-1 and Cys-C kits were all from R\&D Systems, Inc. (Minneapolis, MN, USA). Enzyme-labeled instrument was purchased Bio-Tek ELx800 (Bio-Tek Instruments, Inc. Winooski, VT, USA). The centrifuge used was purchased from Beckman Coulter (Brea, CA, USA). Fully automatic chemistry analyzer was used to detect Scr, Hitachi 7170A (Hitachi, Tokyo, Japan).

Operations were strictly carried out as per the manufacturer's instructions. Instructions included compounding standard samples, adding specimen, incubating, allocating transfusion, washing, adding enzyme, incubating, washing, developing color, terminating, determining, drawing standard curve with curve expert 1.3 using concentration of standard samples as ordinates and corresponding optical density (OD) values as abscissa, calculating curve equation, and then obtaining actual concentration of samples to be detected through substituting OD values to equation to obtain concentration of each sample which was used to multiply dilution ratio. eGFR was calculated using simplified MDRD software (Germantown,MD, USA) (https://www.niddkrepository.org/studies/mdrd/).

Statistical analysis. We analyzed data using SPSS 19.0 statistical software (SPSS, Inc., Chicago, IL, USA). Measurement data were indicated as mean \pm standard deviation. We compared among groups using one-way ANOVA and countable data were presented as samples or percentage. The $\chi^{2}$ test was used to compare among groups. Analysis of diagnostic susceptibility and specificity of NGAL, KIM-1 and Cys-C were realized using receiver operating characteristic curve (ROC). $\mathrm{P}<0.05$ was considered to indicate a statistically significant difference.

\section{Results}

Comparisons of observation index of each group. NGAL, KIM-1 and Cys-C levels in the group of asphyxiant renal injury at 24 and $48 \mathrm{~h}$ were markedly higher than the other two groups $(\mathrm{P}<0.05)$. Changes in $\mathrm{Scr}$ and eGFR within $24 \mathrm{~h}$ were not significant $(\mathrm{P}>0.05)$. Scr level in the renal injury group in $48 \mathrm{~h}$ was significantly elevated while eGFR level decreased considerably $(\mathrm{P}<0.05)$ (Table I).

Analysis of NGAL, KIM-1 and Cys-C. Area under the curve (AUC) for NGAL in the group of asphyxiant renal injury at 24 and $48 \mathrm{~h}$ was significantly higher than KIM-1 and Cys-C. Susceptibility and specificity was improved (Table II, and Figs. 1 and 2).

\section{Discussion}

Compared with glomerulus, proximal tubular in premature infants has poorer development, reabsorption and its ability to treat water-electrolyte as well as acid-base balance is seriously undermined. Compared to mature infants, premature infants with lower gestational age and lower birth weight suffer from lower rate of nephron and lower glomerular filtration. In premature infants, GFR that influence renal vasoconstriction and relaxation is usually low. Thus AKI is more likely to happen in premature infant (7-9).

NGAL is involved in the formation and reparation of renal tubular epithelial cells. It has been established that NGAL level is high in proximal tubular epithelial cells and in the case of AKI, renal tubular epithelial cells are damaged and NGAL levels in blood and urine is very high (10).

Devarajan showed that in renal ischemia reperfusion injury model in rat, NGAL could be detected when AKI occurs for $2 \mathrm{~h}$. However, at the same time, Scr level remained at its normal level. It was concluded that compared with Scr, 
Table II. Analysis of NGAL, KIM-1 and Cys-C.

\begin{tabular}{|c|c|c|c|c|c|c|c|c|c|c|}
\hline & $\begin{array}{l}24 \mathrm{~h} \\
\text { AUC }\end{array}$ & $95 \% \mathrm{CI}$ & $\begin{array}{c}\text { Susceptibility } \\
(\%)\end{array}$ & $\begin{array}{c}\text { Specificity } \\
(\%)\end{array}$ & Cut-off & $\begin{array}{l}48 \mathrm{~h} \\
\mathrm{AUC}\end{array}$ & $95 \%$ CI & $\begin{array}{c}\text { Susceptibility } \\
(\%)\end{array}$ & $\begin{array}{c}\text { Specificity } \\
(\%)\end{array}$ & Cut-off \\
\hline NGAL & 0.836 & $0.634-0.947$ & 86.4 & 83.7 & $48.5 \mathrm{ng} / \mathrm{ml}$ & 0.892 & $0.714-0.968$ & 87.5 & 84.3 & $123.7 \mathrm{ng} / \mathrm{ml}$ \\
\hline KIM-1 & 0.812 & $0.526-0.936$ & 82.3 & 76.7 & $27.4 \mathrm{ng} / \mathrm{l}$ & 0.856 & $0.587-0.942$ & 84.5 & 79.8 & $66.5 \mathrm{ng} / 1$ \\
\hline Cys-C & 0.803 & $0.475-0.921$ & 81.4 & 75.3 & $18.6 \mathrm{ng} / \mathrm{mg}$ & 0.844 & $0.496-0.939$ & 82.2 & 77.9 & $58.9 \mathrm{ng} / \mathrm{mg}$ \\
\hline
\end{tabular}

AUC, area under curve; NGAL, neutrophil gelatinase-associated lipocalin; KIM-1, kidney injury molecule-1; Cys-C, cystatin C.

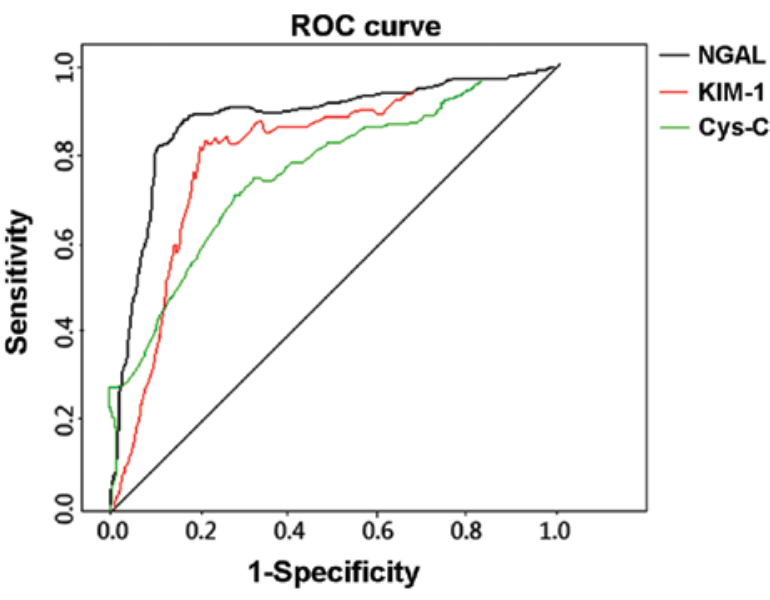

Figure 1. Receiver operating characteristic (ROC) curves of NGAL, KIM-1 and Cys-C in asphyxiant renal injury at $24 \mathrm{~h}$. NGAL, neutrophil gelatinaseassociated lipocalin; KIM-1, kidney injury molecule-1; Cys-C, cystatin C.

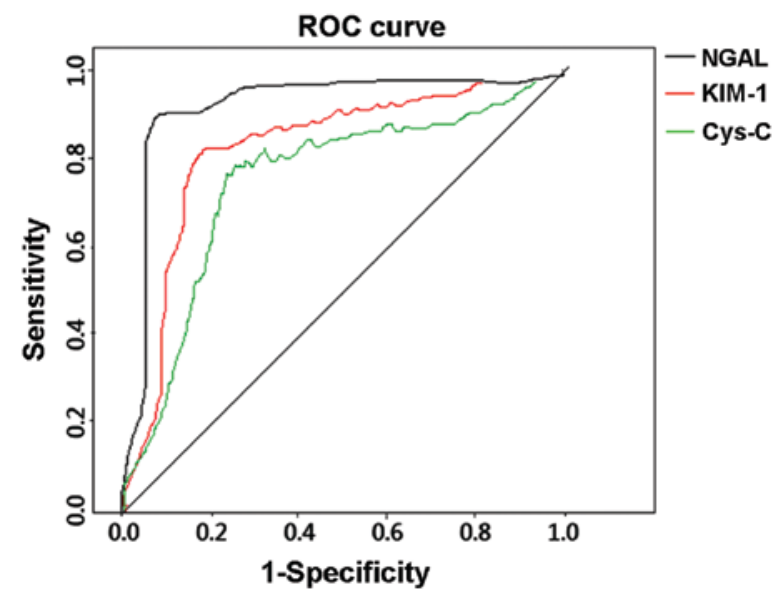

Figure 2. Receiver operating characteristic (ROC) curves of NGAL, KIM-1 and Cys-C in asphyxiant renal injury at $48 \mathrm{~h}$. NGAL, neutrophil gelatinaseassociated lipocalin; KIM-1, kidney injury molecule-1; Cys-C, cystatin C.
NGAL was more sensitive and a better marker for early detection of AKI (11).

Results obtained by Yavuz et al on 22 cases of burned children who were admitted to hospital within $12 \mathrm{~h}$ revealed that there were no significant differences between the Scr level in the 1-5 days AKI group and that in the non-AKI group. By contrast, blood and urine NGAL levels increased considerably in the AKI group which indicated the sensitivity of NGAL for early detection of AKI (12).

Results obtained from another related study on 60 cases of neonates in critical condition showed that NGAL level had little to do with sepsis (13). In that study, according to diagnostic code of AKIT, the cases were divided into the AKI group and the non-AKI group. The results of that study revealed that, the blood NGAL level markedly increased in the early stages of AKI. It was shown that when the NGAL level in blood surpassed the $117.5 \mathrm{ng} / \mathrm{ml}$ mark it was an indication of AKI. Susceptibility was $82.0 \%$ and specificity was $88.5 \%$ (13).

The results presented by Askenazi et al demonstrated that the baseline assessment of urine NGAL in premature infant and gestational age were negatively correlated, while an elevated level of urine NGAL and the degree of renal injury were positively correlated (14).

KIM-1 is a member of the immunoglobulin superfamily. KIM-1 is a transmembrane glycoprotein which comprised mucoprotein and immunoglobulin. It usually engages in recovering process of renal tubule epithelial cells, renal interstitial fibrosis and immunological reactions. It has the functions of adhering and cleaning up the apoptotic cells (15). Under normal physiological conditions, KIM-1 expression level in normal kidney tissue fluctuates between zero to very low. In a case of AKI caused by ischemia or toxic kidney injury, KIM-1 expression level in kidney tissue was markedly increased (16). When proximal tubular injury occurs, extracellular KIM-1 enters into urine in the form of soluble fragment which is relatively stable and easy to detect (17).

Cys- $\mathrm{C}$ is a tyep of cystatin, which is produced by all the karyocytes in the body. It is a non-glycosylated alkaline protein with a low molecular weight that can only be cleaned up by passing through glomerular filtration. It is reabsorbed in proximal convoluted tubule, then decomposed totally and metabolized. It is an endogenous marker that reflects GFR (18).

We showed that NGAL, KIM-1 and Cys-C levels in the group of asphyxiant renal injury within 24 and $48 \mathrm{~h}$ were markedly higher than the two other groups. Changes in Scr and eGFR within $24 \mathrm{~h}$ were not significant. Scr of renal injury within 48 h was markedly elevated while eGFR reduced obviously. Our results indicated that urine NGAL, KIM-1 and Cys- $\mathrm{C}$ could respond to AKI in the early stage. Compared with KIM-1 and Cys-C, AUC of NGAL in the group of asphyxiant renal injury within 24 and 48 h was obviously higher and both susceptibility and specificity was improved. Results also suggested that urine NGAL may have higher diagnostic accuracy compared with KIM-1 and Cys-C.

In conclusion, the critical value of detecting urine NGAL in renal injury in asphyxia preterm infants at $24 \mathrm{~h}$ was $48.5 \mathrm{ng} / \mathrm{ml}$, 
susceptibility was $86.4 \%$ and specificity was $83.7 \%$. The critical value of detecting urine NGAL in renal injury in asphyxia preterm infants within $48 \mathrm{~h}$ was $123.7 \mathrm{ng} / \mathrm{ml}$, susceptibility was $87.5 \%$ and specificity was $84.3 \%$.

\section{References}

1. Beck S, Wojdyla D, Say L, Betran AP, Merialdi M, Requejo JH, Rubens C, Menon R and Van Look PF: The worldwide incidence of preterm birth: a systematic review of maternal mortality and morbidity. Bull World Health Organ 88: 31-38, 2010.

2. Ronco C, Levin A, Warnock DG, Mehta R, Kellum JA, Shah S and Molitoris BA; AKIN Working Group: Improving outcomes from acute kidney injury (AKI): report on an initiative. Int J Artif Organs 30: 373-376, 2007.

3. Sweetman DU and Molloy EJ: Biomarkers of acute kidney injury in neonatal encephalopathy. Eur J Pediatr 172: 305-316, 2013.

4. Urbschat A, Obermüller N and Haferkamp A: Biomarkers of kidney injury. Biomarkers 16 (Suppl 1): S22-S30, 2011.

5. Peralta CA, Katz R, Bonventre JV, Sabbisetti V, Siscovick D, Sarnak M and Shlipak MG: Associations of urinary levels of kidney injury molecule 1 (KIM-1) and neutrophil gelatinase-associated lipocalin (NGAL) with kidney function decline in the Multi-Ethnic Study of Atherosclerosis (MESA). Am J Kidney Dis 60: 904-911, 2012.

6. Duan SB, Liu GL, Yu ZQ and Pan P: Urinary KIM-1, IL-18 and Cys-c as early predictive biomarkers in gadolinium-based contrast-induced nephropathy in the elderly patients. Clin Nephrol 80: 349-354, 2013.

7. Dagher PC, Herget-Rosenthal S, Ruehm SG, Jo SK, Star RA, Agarwal R and Molitoris BA: Newly developed techniques to study and diagnose acute renal failure. J Am Soc Nephrol 14 2188-2198, 2003

8. Sutherland MR, Gubhaju L, Moore L, Kent AL, Dahlstrom JE, Horne RS, Hoy WE, Bertram JF and Black MJ: Accelerated maturation and abnormal morphology in the preterm neonatal kidney. J Am Soc Nephrol 22: 1365-1374, 2011.
9. Walker MW, Clark RH and Spitzer AR: Elevation in plasma creatinine and renal failure in premature neonates without major anomalies: terminology, occurrence and factors associated with increased risk. J Perinatol 31: 199-205, 2011.

10. Peco-Antić A, Ivanišević I, Vulićević I, Kotur-Stevuljević J, Ilić S, Ivanišević J, Miljković M and Kocev N: Biomarkers of acute kidney injury in pediatric cardiac surgery. Clin Biochem 46: 1244-1251, 2013

11. Devarajan P: Neutrophil gelatinase-associated lipocalin (NGAL): a new marker of kidney disease. Scand J Clin Lab Invest Suppl 241: 89-94, 2008

12. Yavuz S, Anarat A, Acartürk S, Dalay AC, Kesiktaş E, Yavuz M and Acartürk TO: Neutrophil gelatinase associated lipocalin as an indicator of acute kidney injury and inflammation in burned children. Burns 40: 648-654, 2014.

13. El-Farghali OG, El-Raggal NM, Mahmoud NH and Zaina GA: Serum neutrophil gelatinase-associated lipocalin as a predictor of acute kidney injury in critically-ill neonates. Pak J Biol Sci 15 : 231-237, 2012.

14. Askenazi DJ,Koralkar R,Levitan EB, Goldstein SL,Devarajan P, Khandrika S, Mehta RL and Ambalavanan N: Baseline values of candidate urine acute kidney injury biomarkers vary by gestational age in premature infants. Pediatr Res 70: 302-306, 2011.

15. Kirk R: Renal fibrosis: KIM-1 expression links kidney injury with CKD in mice. Nat Rev Nephrol 9: 627, 2013.

16. Cruz DN, Goh CY, Haase-Fielitz A, Ronco C and Haase M: Early biomarkers of renal injury. Congest Heart Fail 16 (Suppl 1): S25-S31, 2010.

17. Sabbisetti VS, Ito K, Wang C, Yang L, Mefferd SC and Bonventre JV: Novel assays for detection of urinary KIM-1 in mouse models of kidney injury. Toxicol Sci 131: 13-25, 2013.

18. Dorum S, Silfeler I, Dorum BA, Silfeler DB, Canbak Y and Say A: Reference values of serum cystatin-C for full-term and preterm neonates in Istanbul. Indian J Pediatr 79: 1037-1042, 2012. 